Supplement of Nonlin. Processes Geophys., 21, 971-985, 2014

http://www.nonlin-processes-geophys.net/21/971/2014/

doi:10.5194/npg-21-971-2014-supplement

(C) Author(s) 2014. CC Attribution 3.0 License.

(c) (i)

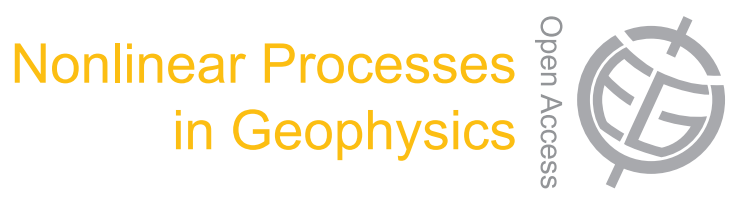

Supplement of

\title{
Representing model error in ensemble data assimilation
}

C. Cardinali et al.

Correspondence to: C. Cardinali (carla.cardinali@ecmwf.int) 
We summarize the basic steps and properties of the 3D orthogonal normal modes derived in Kasahara and Puri (1981, KP1981 hereafter). This summary closely follows notes by Kasahara (2014, monograph in preparation, K2014 hereafter). Then we use it to show that the ensemble variance computed in the modal space corresponds to the total variance of the wind and geopotential fields in physical space.

\section{Projection of $3 \mathrm{D}$ model-level data onto normal-mode functions}

The normal-mode function (NMF) expansion of 3D data assumes that solutions to the adiabatic and inviscid linearized equations at time $t$ are characterized by the separability of the vertical and horizontal dependences of the dependent variables. The NMF derivation in KP1981 introduced a geopotential variable $P$ defined as $P=\Phi+R T_{o} \ln \left(p_{s}\right)$ which allowed derivation of 3D orthogonal NMF. All parameters here have their usual meaning; i.e. $\Phi=g h$ is the geopotential, $p_{s}$ is the surface pressure, the globally averaged temperature on model levels is denoted by $T_{o}, R$ is the gas constant and $g$ is gravity.

A discrete input dataset of the zonal and meridional winds $(u, v)$ and geopotential height $h=P / g$ at time step $t$ is defined on the horizontal regular Gaussian grid and vertical $\sigma$ levels. The projection is performed on the precomputed vertical structure functions $\Pi$, the horizontal Hough vector functions in the meridional direction and waves in the longitudinal direction. For a detailed derivation, discussion of the Hough functions and treatment of the zonally average state, the reader is referred to KP1981 and references therein as well as K2014. Here we simply make use of the orthogonality relationships derived in those papers.

The procedure is summarized as follows. Input data on $j$-th $\sigma$ level are first represented by a series of the vertical structure function $\Pi_{m}(j)$. For a single data point $\left(\lambda, \varphi, \sigma_{j}\right)$ the expansion is

$$
\left|\begin{array}{l}
u\left(\lambda, \varphi, \sigma_{j}\right) \\
v\left(\lambda, \varphi, \sigma_{j}\right) \\
h\left(\lambda, \varphi, \sigma_{j}\right)
\end{array}\right|=\sum_{m=1}^{M} \mathbf{S}_{m} \mathbf{X}_{m}(\lambda, \varphi) \Pi_{m}(j)
$$

The scaling matrix $\mathbf{S}_{m}$ in (1), which makes the vector $\mathbf{X}_{m}(\lambda, \varphi)$ for the input to the horizontal structure equation dimensionless, is defined as

$$
\left[\begin{array}{ccc}
\sqrt{g D_{m}} & 0 & 0 \\
0 & \sqrt{g D_{m}} & 0 \\
0 & 0 & D_{m}
\end{array}\right]
$$

The integer subscript $m$ identifies the vertical mode which spans from the external mode $m=1$ to the total number of vertical modes $M$. Functions $\Pi_{m}(j)$ defined for the finite difference method for the $\sigma$ vertical levels are orthogonal (KP1981):

$$
\sum_{j=1}^{J} \Pi_{m}(j) \Pi_{m^{\prime}}(j)=\delta_{m m^{\prime}}
$$

Here, $J$ is the number of vertical levels. 
The horizontal coefficient vector $\mathbf{X}_{m}(\lambda, \varphi)$ is calculated by the reverse transform of (1) through multiplication of (1) by $\Pi_{m^{\prime}}(j)$ and summation of the result from $j=1$ to $J$ with the use of the orthogonality condition (2). The result becomes

$$
\mathbf{X}_{m}(\lambda, \varphi)=\mathbf{S}_{m}^{-1} \sum_{j=1}^{J}(u, v, h)^{\mathrm{T}} \Pi_{m}(j)
$$

Equations (1) and (3) are the vertical transform pair.

The horizontal coefficient vector $\mathbf{X}_{m}(\lambda, \varphi)$ for a given vertical mode $m$ can now be projected onto the Hough harmonics defined as

$$
\mathbf{H}_{k}^{r}(\lambda, \varphi ; m)=\mathbf{\Theta}_{k}^{r}(\varphi ; m) e^{\mathrm{i} k \lambda} .
$$

In this equation, we use the subscript $r$ for the meridional mode to indicate all combined meridional normal modes including rotational (ROT), and eastward and westward propagating inertio-gravity (EIG and WIG) modes. The subscript $r$ is thus a tag index ranging from 1 to integer $R$ defined by

$$
R=N_{R}+N_{W I G}+N_{E I G}
$$

where ROT mode: $n_{R}=0,1,2, \ldots, N_{R}-1$, EIG mode: $n_{E I G}=0,1,2, \ldots, N_{E I G}-1$, WIG mode: $n_{W I G}=0,1,2, \ldots, N_{W I G}-1$. The Hough harmonics are therefore characterized by the two indices for combined meridional mode $r$ and the zonal wavenumber $k$ for every given vertical mode $m$.

The horizontal coefficient vector $\mathbf{X}_{m}(\lambda, \varphi)$ for a vertical mode $m$ is projected onto the Hough harmonics as

$$
\mathbf{X}_{m}(\lambda, \varphi)=\sum_{r=1}^{R} \sum_{k=-K}^{K} \chi_{k}^{r}(m) \mathbf{H}_{k}^{r}(\lambda, \varphi ; m)
$$

where the maximal number of zonal waves is denoted by $K$, including zero for the mean zonal state. Vector $\mathbf{X}_{m}$ is defined for every vertical mode as $\mathbf{X}_{m}(\lambda, \varphi)=\left(u_{m}, v_{m}, h_{m}\right)^{\mathrm{T}}$ following (1).

As discussed in details in K2014 and references therein, the global orthogonality of the Hough functions written as:

$$
\frac{1}{2 \pi} \int_{0}^{2 \pi} \int_{-1}^{1} \mathbf{H}_{k}^{r} \cdot\left[\mathbf{H}_{k^{\prime}}^{r^{\prime}}\right]^{*} d \mu d \lambda=\delta_{k k^{\prime}} \delta_{r r^{\prime}},
$$

applies with the global inner product defined as

$$
<\mathbf{W}_{i}, \mathbf{W}_{j}^{*}>=\frac{1}{2 \pi} \int_{0}^{2 \pi} \int_{-1}^{1}\left(\tilde{u}_{i} \tilde{u}_{j}^{*}+\tilde{v}_{i} \tilde{v}_{j}^{*}+\tilde{h}_{i} \tilde{h}_{j}^{*}\right) d \mu d \lambda .
$$

Here, $\mu=\sin (\varphi)$, and subscripts $i$ and $j$ correspond to two modal indices, each defined by a combination of the zonal wavenumber $k$ and the meridional mode $r$. Vector $\mathbf{W}=$ 
$\left(\tilde{u}_{i}, \tilde{v}_{i}, \tilde{h}_{i}\right)^{\mathrm{T}}=\left(u_{m} / \sqrt{g D_{m}}, v_{m} / \sqrt{g D_{m}}, h_{m} / D\right)^{\mathrm{T}}$ contains now non-dimensional variables describing shallow-water motions for every equivalent depth $D_{m}$.

The scalar complex coefficient $\chi_{k}^{r}(m)$ can be obtained from (6) by multiplying (6) by $\left[\mathbf{H}_{r}^{k}\right]^{*}$, the complex conjugate of $\mathbf{H}_{r}^{k}$, and integrating the resultant equation with respect to $\lambda$ from 0 to $2 \pi$, and with respect to $\varphi$ from $-\pi / 2$ to $+\pi / 2$, and using the orthonormality condition (7). The result is

$$
\chi_{k}^{r}(m)=\frac{1}{2 \pi} \int_{0}^{2 \pi} \int_{-1}^{1} \mathbf{X}_{m}(\lambda, \varphi) \cdot\left[\mathbf{H}_{r}^{k}\right]^{*} d \mu d \lambda .
$$

Equations (6) and (9) are the horizontal transform pair.

\section{Application of NMF expansion to the ECMWF ensemble and computa- tion of the ensemble spread}

Dynamical variance of the ECMWF ensemble is defined for a 3D baroclinic atmosphere transformed to the $M$ layers of shallow-water system $(m=1, \ldots, M$, each with the equivalent depths $D_{m}$ ) by the vertical transform (3).

$$
\sum_{i} \sum_{j} \sum_{m} \Sigma_{p s}^{2}
$$

where $i, j$, and $m$ represent indices in the zonal, meridional and vertical directions, respectively, and variance $\Sigma_{p s}^{2}$ is defined as

$$
\Sigma_{p s}^{2}(i, j, m)=\frac{1}{N-1} \sum_{i=1}^{N}\left(u_{i}^{2}+v_{i}^{2}+\frac{g}{D_{m}} h_{i}^{2}\right) .
$$

Here, $u_{i}, v_{i}$ and $h_{i}$ denote departures of the ensemble member $i$ from the ensemble mean for wind components and geopotential height on $\sigma$ model levels. The size of the ensemble is denoted by $N$. The normalization constant $D_{m}$ is the equivalent height, defined for every vertical mode $m$. The total variance in the modal space is defined as

$$
\sum_{k} \sum_{r} \sum_{m} \Sigma_{m s}^{2}
$$

where variance $\Sigma_{m s}^{2}$ is defined as

$$
\Sigma_{m s}^{2}(k, r, m)=\frac{1}{N-1} \sum_{i=1}^{N} g D_{m}\left(\chi_{k}^{r}(m ; i)\left[\chi_{k}^{r}(m ; i)\right]^{*}\right) .
$$

The variable $h$ in equation (11) is defined in the first section, $h=\frac{P}{g}$. Both modal-space and physical-space variance are in units $\mathrm{ms}^{-1}$ i.e. $\mathrm{J} / \mathrm{kg}$. 
In order to show that equations (10) and (12) provide the same total variance, we write the NMF expansion for deviations of a single ensemble member $i$ from the ensemble mean as

$$
\chi_{k}^{r}(m ; i)=\frac{1}{2 \pi} \int_{0}^{2 \pi} \int_{-1}^{1}\left(\mathbf{S}_{m}^{-1} \sum_{j=1}^{J}\left(u_{i}, v_{i}, h_{i}\right)^{\mathrm{T}} \Pi_{m}(j)\right) \cdot\left[\mathbf{H}_{r}^{k}\right]^{*} d \mu d \lambda
$$

First we multiply equation (14) by $\mathbf{H}_{k}^{r}$, integrate the result with respect to $\lambda$ from 0 to $2 \pi$, and with respect to $\varphi$ from $-\pi / 2$ to $+\pi / 2$, and use the orthonormality condition ( 7 ) to get the following expression:

$$
\frac{1}{2 \pi} \int_{0}^{2 \pi} \int_{-1}^{1} \chi_{k}^{r}(m ; i) \mathbf{H}_{k}^{r} d \mu d \lambda=\mathbf{S}_{m}^{-1} \sum_{j=1}^{J}\left(u_{i}, v_{i}, h_{i}\right)^{\mathrm{T}} \Pi_{m}(j) .
$$

Now we multiply (15) by $\Pi_{m^{\prime}}(j)$ and sum over all levels with the use of (2) to obtain

$$
\frac{1}{2 \pi} \int_{0}^{2 \pi} \int_{-1}^{1} \sum_{j=1}^{J} \chi_{k}^{r}(m ; i) \mathbf{H}_{k}^{r} \Pi_{m}(j) d \mu d \lambda=\mathbf{S}_{m}^{-1}\left(u_{i}, v_{i}, h_{i}\right)^{\mathrm{T}} .
$$

Finally, multiplying (16) by $g D_{m}\left(u_{i}, v_{i}, h_{i}\right) \mathbf{S}_{m}$, and noticing that

$$
\left[\chi_{k}^{r}(m ; i)\right]^{*}=\frac{1}{2 \pi} \int_{0}^{2 \pi} \int_{-1}^{1} \mathbf{H}_{r}^{k} \mathbf{S}_{m}^{-1} \sum_{j=1}^{J}\left(u_{i}, v_{i}, h_{i}\right) \Pi_{m}(j) d \mu d \lambda,
$$

the left-hand side of equation (16) becomes

$$
g D_{m} \chi_{k}^{r}(m ; i)\left[\chi_{k}^{r}(m ; i)\right]^{*}
$$

the modal-space variance for mode $(k, r, m)$. The right hand side correspond to

$$
g D_{m}\left(\left(u_{i}, v_{i}, h_{i}\right) \mathbf{S}_{m}^{-2}\left(u_{i}, v_{i}, h_{i}\right)^{\mathrm{T}}\right)=u_{i}^{2}+v_{i}^{2}+\frac{g}{D_{m}} h_{i}^{2} .
$$

After averaging (18) over the ensemble and summing over the whole modal space, we obtain the total variance defined by equation (12) corresponding to the sum of the $u, v$, and $h$ variances in the physical space as defined by equation (10). If we need to refer to the model $(\sigma)$ levels instead of vertical modes, we need to apply transformation (1).

\section{References:}

Kasahara, A. and K. Puri, 1981: Spectral representation of three-dimensional global data by expansion in normal mode functions, Mon. Wea. rev., 109, 37-51.

Kasahara, A., 2014: Normal-mode function expansion. Monograph. Available on request. 